\title{
The effect of Gunn's intramuscular stimulation for postherpetic neuralgia -A report of 4 cases-
}

\author{
Wook Jung, Byung-Jun Lee, Sin Sung Kim, and Young Jin Lee \\ Department of Anesthesiology and Pain Medicine, Donkang Hospital, Ulsan, Korea
}

Herpes zoster is the consequence of reactivation of latent varicella zoster virus from dorsal root ganglia. Postherpetic neuralgia (PHN) may be diagnosed when pain persists in a dermatomal pattern long after the vesicular erruption has healed. PHN is a kind of neuropathic pain. The pathophysiology of PHN is uncertain, but neuropathic pain due to denervation supersensitivity may be important to understand the pathophysiology of PHN. Numerous treatment have been introduced for the management of PHN, but no methods that results in complete remission. Gunn's intramuscular stimulation (IMS) is one of the best treatment of chronic pain, especially neuropathic pain. We tried Gunn's IMS for treatment of PHN patients affecting thoracic dermatomes. As a result, the visual analogue scale (VAS) was decreased from 7-8 to $2-3$ and the result were satisfactory. The purpose of this case report is to introduce the Gunn's IMS and review our experience for the treatment of PHN. (Korean J Anesthesiol 2010; 58: 311-317)

Key Words: Gunn's IMS, Neuropathic pain, Postherpetic neuralgia.

Varicella zoster occurs as a result of the infections with varicella-zoster virus, and it is latently present in the dorsal root ganglion of spinal cord. Then, it is re-activated due to the impaired or suppressed immune responses. In the skin areas where the corresponding nerves are innervated, it produces a unilateral, vesicular eruption. In addition to the skin symptoms, the pain is concurrently present at the site of invasion. Besides, the skin is not healed but can be progressed to postherpetic neuralgia (PHN). Postherpetic neuralgia (PHN) varicella zoster is a pain which is persistently present for more than 30 days following the onset of skin rash or for more than 3 months [1].
Infections with varicella zoster virus trigger the occurrence of inflammations in the skin tissue and then stimulate the sympathetic nervous system. Thus, they cause the decreased perfusion of carpillary vessels within the nerve fibers [2]. This decreased blood flow creates an ischemia. A persistent presence of the ischemia causes the degeneration of nerve transmission fibers. Owing to this, patients complain of persistent pain, allodynia or paresthesia. In severe cases, there might be such symptoms as depression, insomnia and decreased appetite. In these cases, there is a difficulty of maintaining the daily lives.

Most cases of varicella zoster are healed without compli-

Received: July 6, 2009. Revised: 1st, July 16, 2009; 2nd, September 13, 2009. Accepted: October $19,2009$.

Corresponding author: Byung-Jun Lee, M.D., Department of Anesthesiology and Pain Medicine, Donkang Hospital, Taehwa-dong, Jung-gu, Ulsan 681-711, Korea. Tel: 82-52-241-1297, Fax: 82-52-241-1295, E-mail: bowfamily@naver.com

(c) This is an open-access article distributed under the terms of the Creative Commons Attribution Non-Commercial License (http:// creativecommons.org/licenses/by-nc/3.0/), which permits unrestricted non-commercial use, distribution, and reproduction in any medium, provided the original work is properly cited. 
cations. In approximately $10 \%$ of total cases, however, the disease is progressed to neuralgia following varicella zoster. In elderly patients aged 60 years or older, those with diabetes mellitus or immunocompromised ones, the morbidity has been reported to be increased.

It is an intractable disease for which there are no established treatment regimens. For the treatment, however, there are treatment methods such as drug therapy (anti-depressants or anti-convulsants) and local swabbing anesthetics or nerve block. In particular, epidural block or intercostal nerve block are advantageous in accurately treating the affected dermatomes. Therefore, these treatment regimens are frequently performed at many pain clinics.

An injection for sensitized and chronic lesions (trigger point injection) or dry needling have been widely accepted as the effective treatment regimens for pain control. But Gunn further systematized these regimens and then considered the deep paravertebral muscles and the stimulation of vertebral joints to be important. Then, this was termed as deep dry needling or Gunn's IMS. Based on these treatment regimens, interventional microadhesilolysis and nerve stimulation (IMNS) and fluoroscopy guided interventional musculoskeletal adhesiolysis and nerve stimulation (FIMS) have been developed to provide more invasive and powerful stimulations. These treatment modalities are effective for the treatment of hypersensitized neuropathy due to the damage of peripheral nerve, one of the mechanisms by which the chronic pain is generated.

Given the above background, we experienced good treatment outcomes using Gunn's IMS treatment in a case of neuropathy following varicella zoster infections. Here, we report our case with a review of literatures.

\section{Case Report}

\section{Case 1}

A 53-year-old female patient visited us with a chief complaint of sharp and burning pain developed in the right chest. Six months prior to outpatient visit, due to the presence of varicella zoster occurring in the $5^{\text {th }}$ and $6^{\text {th }}$ dermatome of the right chest, the patient visited department of dermatology. Then, the patient continually received the treatments using anti-viral agents, non-steroidal anti-inflammatory agents, anti-depressants and digestive drugs. Due to the persistent presence of pain, however, the patient was referred to a pain clinic of the current medical institution. On physical examination, in the $5^{\text {th }}$ and $6^{\text {th }}$ dermatomes of right chest areas, the patient presented with the aggravated nocturnal allodynia and hyperesthesia. In this patient, the visual analogue scale (VAS) was $7 / 10$. The patient complained of severe pain. On baseline examination, there were no notable findings.

For the treatment, a flexible needle which was specifically designed for IMS therapy, of $0.25 \mathrm{~mm}$ in thickness and $50 \mathrm{~mm}$ in length, was inserted in a plunger (Fig. 1). A needle was thin and easily flexible. Accordingly, it can minimize risks of developing the damage of vital blood vessels or nerve tissue. In the areas which were $1 \mathrm{~cm}$ lateral to the spinou process of the $6^{\text {th }}$ and $7^{\text {th }}$ thoracic vertebra, a needle was placed vertical to the skin until the bone contact was perceived. Then, a needle was rotated several times and thereby the multifidus muscle was stimulated. In the areas which were $3-4 \mathrm{~cm}$ lateral to the intervertebral space at such levels as $\mathrm{T} 5-6, \mathrm{~T} 6-7$ and $\mathrm{T} 7-8$, to prevent the occurrence of pneumothorax, a needle was placed in a slanted manner at an angle of 15-20 degree from the vertical line to the vertebral directions. Then, a needle was rotated several times. Thus, the tissue around the vertebral facet was stimulated. T5 -7 thoracic dermatomes were stimulated (Fig. 2). During Gunn's IMS therapy, no attempts of pharmacological injections have been made excluding a needle. Because a needle stimulation was sufficient for the tissue around the multifidus muscle originating from the spinous process and inserting in the caudal facet joint and the tissue around the posterior facet where the medial branch of primary dorsal ramus was distributed, as shown in medial branch block, without using such equipments as fluoroscopy or ultrasonography, the thoracic spinous process at the affected spinal segment was selected as an anomical landmark. Then, without any complications, the surgery was successfully performed. During the first two weeks, the treatment was performed twice a week at a 3-day interval. During the following two weeks, the treatment was performed six times once a week for a total of four weeks.

Four weeks following the treatment, VAS was improved from 7 to 3 points. An examination was performed after two weeks,

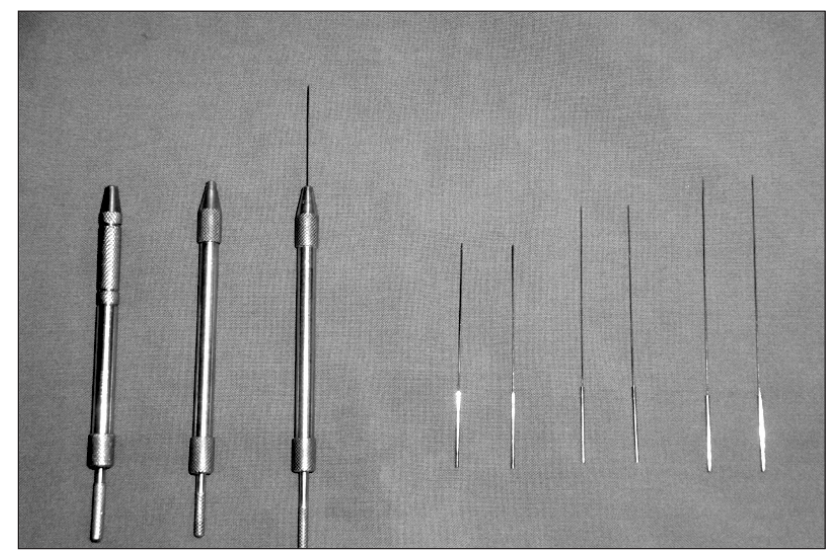

Fig. 1. This photograph shows that various length of IMS needles (Right) and types of plungers (Left). 


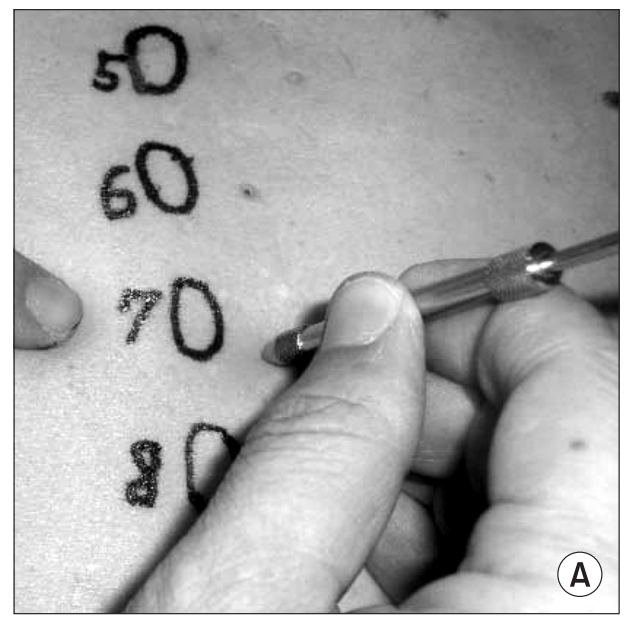

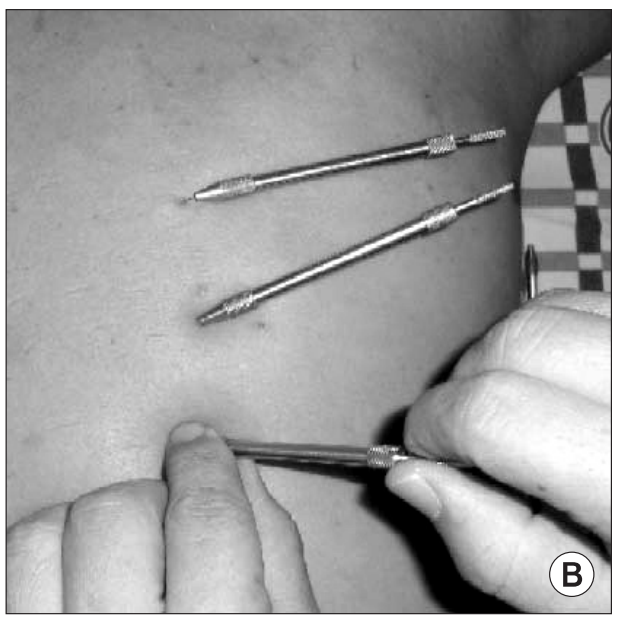

Fig. 2. (A) This photograph shows that anatomic landmark when Gunn's IMS treatment has undergone to thoracic multifidi (5-8th). (B) This photograph shows that thoracic facet joints (5-7th) stimulation has undergone with plunger. and this showed that VAS was maintained at 3 points. Then, the treatment was completed.

\section{Case 2}

A 66-year-old woman visited us with a chief complaint of intermittent pin-prick pain occurring in the right chest. During a 4-month period, due to the occurrence of neuralgia developed varicella zoster which occurred along the the $4^{\text {th }}$ thoracic dermatome, the patient underwent intermittent stellate ganglion block and intercostal nerve block at a local clinic. Meanwhile, the symptoms were not improved. Therefore, the patient was admitted to our medical institution. The patient stated that the symptoms were particularly worsened during the night, who were prescribed to take analgesics and antidepressants. Three weeks prior to outpatient visit, the patient deliberately discontinued the administration of drugs. On physical examination, the patient complained of allodynia and hyperesthesia where there was a concurrent presence of the scar tissue formed in the corresponding dermatomes. At the time of admission, VAS was $7-8 / 10$ points. For the treatment, a needle was placed in the multifidus muscle at the level of T5 and the tissue around the facet at the level of T5-6. Four weeks after the treatment, VAS was decreased from 7-8 points at the time of admission to 3 points. Two weeks later, according to an evaluation performed in an outpatient setting, VAS was improved to 2 points and this led to a closure of the treatment.

\section{Case 3}

A 57-year-old man visited us with a chief complaint of stabbing pain occurring in the left chest. Four months prior to outpatient visit, due to the presentation of skin lesions and pain occurring in the left $7^{\text {th }}$ and $8^{\text {th }}$ thoracic vertebra, the patient was diagnosed with varicella zoster at department of dermatology and then received the drug treatments. But the pain was persistent present. At a local clinic, the patient was diagnosed with neuralgia following varicella zoster. Then, the patient received subcutaneous drug treatment and intercostal nerve block. Due to a lack of the improvement of symptoms, however, the patient was referred to us. On physical examination, in the left $7^{\text {th }}$ and $8^{\text {th }}$ thoracic dermatomes, there were allodynia and hyperesthesia. VAS was $7-8 / 10$ points. For the treatment, a needle was placed in the thoracic $8^{\text {th }}$ and $9^{\text {th }}$ multifidus muscle and thoracic $7^{\text {th }}$ and $8^{\text {th }}, 8^{\text {th }}$ and $9^{\text {th }}$ and $9^{\text {th }}$ and $10^{\text {th }}$ tissue around the vertebral facet. Four weeks following the treatment, VAS was decreased from 7-8 point at the time of admission to 3 points. An examination was performed two weeks later, and this showed that VAS was maintained at $2-3$ points and this led to a closure of the treatment.

\section{Case 4}

A 72-year-old man visited us with a chief complaint of intermittent intractable pain occurring in the right chest. On past history, the patient sustained a fracture of the left femur. At department of orthopedic surgery of our medical institution, the patient was hospitalized to take the surgery. At the time of admission, due to the presentation of varicella zoster and its resulting pain extensivly occurring along the right $6-8^{\text {th }}$ dermatomes, the patient received drug treatments at department of dermatology. During the hospitalization treatments, the symptoms were improved. Two months following the discharge, the patient complained of severe pain occurring in the corresponding dermatomes. Then, the patient visited department of dermatology and was referred to a pain clinic. On physical examination, the patient complained of allodynia, hyperesthesia and hyperalgesia where there was a 
concurrent presence of the scar tissue formed in the right $6^{\text {th }}$, $7^{\text {th }}$ and $8^{\text {th }}$ dermatomes. Besides, the patient also complained of sleep disturbance. At the time of admission, VAS was 8/10 points. On routine lab test, there were no notable findings that impede the treatments for diabetes mellitus, blood coaguation disorder and hemostasis abnormality. For the treatment, a needle was placed in the thoracic $8^{\text {th }}$ and $9^{\text {th }}$ multifidus muscle and thoracic $7^{\text {th }}$ and $8^{\text {th }}, 8^{\text {th }}$ and $9^{\text {th }}$ and $9^{\text {th }}$ and $10^{\text {th }}$ tissue around the vertebral facet. Four weeks following the treatment, VAS was decreased from 8 points at the time of admission to 2 points. An examination was performed two weeks later, and this showed that VAS was maintained at 2 points and this led to a closure of the treatment.

In the above patients, the treatment period and frequency were four weeks and a total of six times. Based on the characteristics of patients, the age, sex, disease duration, the pattern of pain and the affected dermatomes showed the different characteristics (Table 1). Following the treatment, VAS improvement rate (VAS improvement rate $(\%)=$ VAS prior to treatment - VAS during evaluation $\times 100$ ) was almost consistent (Fig. 1).

\section{Discussion}

Neuralgia following varicella zoster is defined as the pain which is persistently present even following the treatment of skin lesions. In most cases, with the time progression or after one month, there is a tendency that neuralgia is decreased. Up to a period of $2-6$ months, the disease duration is considered. Regarding the occurrence frequency, approximately $10 \%$ of patients with varicella zoster are progressed to PHN. In elderly patients, those with immunocompromised status or immunosuppressive patients, the morbidity has been reported to reach $20-50 \%$. The affected dermatomes may be some part of body areas. Approxmately more than $50 \%$ of patients develop the lesions in the areas where the thoracic nerves are innervated. Then, in cases of cervical nerve innervation and cranial nerves, the lesions occur the most frequently in the first branch of cranial nerve III. In regard to the skin symptoms, the vesicles are formed with the beginning of rash formation. In the areas where the nerve was invaded, the pain is generated. The pain disappears while the skin symptoms are healed. In some cases, however, the lesions are progressed to PHN and these lesions are persistently present for several months or several years. It is commonly encountered that several symptoms which cannot be verbally expressed. The pain is persistently present and causes a torment. In most cases, it causes a sleep disturbance and the impaired daily lives. Little is known about its pathophysiology. Due to the re-activated varicella zoster virus, the inflammation within the nerve causes the nerve damage. This damage is spreaded to the spinal dorsal root, dorsal root ganglion, peripheral nerves, dorsal nerve root and spinal nerve. Then, it generates an abnormal ectopic electrical potential (peripheral pathogenesis). Following the nerve damage, the sympathetic nerve receptor is increased. In response to the sympathetic nerve stimulation, the sympathetic nerve is sensitively responded (abnormal connection mechanism). Due to the increased activity of nociceptor C-fiber, the excitability of spinal olfactory sensation was increased. Due to the generation of suppressive intermediate neurons, the suppressive mechanisms were impaired. Due to the structural reconstruction of olfactory sensation where the pain is generated due to the normal unharmful stimulations of major myelinated A-beta fibers, the central nervous system was desensitized. It has also theoretically been proposed that the isometric discharge of persistent peripheral nerve fibers triggers the occurrence of central sensitization where NMDA (N-methyl-D-aspartate) plays a major role and this leads to the persistent presence of pain [3-5]. All of these mechanisms are gradually considered to be a key mechanism which causes neuropathic pain. If the detrimental stimulations on the nerve should be terminated during a short-term period, the nerve would immediately make a recovery of the original function. In cases in which the neuralgia is persistently present as shown

Table 1. Characteristics of Patients for Gunn's IMS

\begin{tabular}{|c|c|c|c|c|c|}
\hline Case No. & Sex/Age & Characteristics of pain & Duration (months) & Lesion of dermatome & Treatment site \\
\hline \multirow[t]{2}{*}{1} & $\mathrm{~F} / 53$ & Sharp \& burning & 6 & T5,6 (Rt.) & T6,7 multifidus \\
\hline & & & & & T5-6-7-8 FJ \\
\hline \multirow[t]{2}{*}{2} & $\mathrm{~F} / 66$ & Pin-prick & 4 & T4 (Rt.) & T5 multifidus \\
\hline & & & & & T4-5-6 FJ \\
\hline \multirow[t]{2}{*}{3} & $\mathrm{M} / 57$ & Stabbing & 4 & T7,8 (Lt.) & T8,9 multifidus \\
\hline & & & & & T7-8-9-10 FJ \\
\hline \multirow[t]{2}{*}{4} & $\mathrm{M} / 72$ & Intermittent intractable & 2 & T6,7,8 (Rt.) & $\mathrm{T} 7,8,9$ multifidus \\
\hline & & & & & T6-7-8-9-10 FJ \\
\hline
\end{tabular}

Gunn's IMS: Gunn's intramuscular stimulation, FJ: facet joints. 
in neuralgia following varicella zoster infection, however, the impaired nerve function is persistently present. The sensitvity of peripheral receptors is increased. This may induce the occurrence of chronic pain, i.e., neuropathic pain [6].

Nerve stimulation is one the representative treatment modalities for chronic pain. Exercise or physical therapy are a stimulatory effect via an exercise receptor. A hot pack or massage would have a stimulatory effect on the temperature or baroreceptor. A stimulatory effect of Gunn's IMS therapy induces the electrical damage potential due to a needle of biological tissue. Through a needle, it promotes the releases of chemomediators such as substance P, neurokinin A and tachykinin [7]. Various mechanisms have been used to explain and these mechanisms include the modulation of detrimental afferent information of the central nervous system, the suppression of intercalated cells due to the activation of opioid pain-reducing system, the changes of activity of each cell on the ventral side due to the mechanical, chemical and electrical stimulations or a loss of the denervating response due to a needle $[8,9]$. A stimulatory effect of a needle is maximized when it is close to the nerve root where there is an abundant amount of muscle spindle tissue and the damaged nerve root. The placement of a needle in the deep vertebral muscle, vertebral facet, if possible, or nerve root is the most effective. Following this, a rotation of the needle would further increase the release of chemical mediators as well as the damage current in the muscle spindle tissue or the tissue around the nerve.

In the disease progression from varicella zoster to $\mathrm{PHN}$, the duration extending from the age and rash formation to the initiation of treatment is the most responsible factor. According to Colding, only the sympathetic nerve block which was performed within two weeks following the onset of rash had a preventive effect on the progression to PHN. This author also noted that there was a lack of the effects of sympathetic nerve block in cases of PHN in which the age was increased or more than two months elapsed following the manifestation of symptoms [10]. According to Winnie, in the correlation between the time point of initiation of nerve block treatment and the treatment effect, the treatment prognosis is greatly affected by the age. Particularly in elderly patients, within two months prior to the occurrence of irreversible changes, the sympathetic nerve block is important and the treatment effect is good [11]. This implies that the time point for determining the treatment effect and the initiation of treatment. Accordingly, the use of active treatment in the early stage of varicella zoster infection prevents the progression to PHN. Therefore, this is utmost important. An early administration of anti-viral agents or sympathetic nerve block should be performed for preventive purposes. In the current cases, patients had an age range of $50-70$ years. It can therefore be stated that the age may affect the treatment prognosis. But individual patients receive the treatment without overlooking the symptoms. Besides, the current cases were all recurrences. Due to the delayed initiation of treatment, the morbidity of PHN was increased and this led to the decreased treatment effect, which have been reported by Colding and Winnie, and this was not in agreement with current cases. In the treatment of affected individuals, there are such treatment modalities as anti-depressants, anti-convulsants, opioids, local application, various types of nerve block, other acupunctures, TENS and surgical treatment (a local resection of the affected skin). For oral drug treatments, tricyclic antidepressants have been established as a standard treatment modality. But these drugs are prohibited for the treatment of cardiovascular diseases. That is, there are a substantial number of side effects that have been reported. Such anti-convulsants or opioids as carbamazepine have a greater degree of the side effects as compared with their effectiveness. A novel type of anti-convulsants, gabapentin has a lower profile of the toxicity and side effects and it has been used to treat neuropathic pain. Lee et al. [12] noted that gabapentin has an excellent profile of the treatment effect in patients with PHN. Such treatment modalities as astrocyte ganglion block, epidural nerve block, nerve root block and sympathetic nerve block are also effective treatment methods. In particular, epidural nerve block or intercostal nerve block are advantageous in treating the affected sites both accurately and easily. Therefore, they have frequently been used for the treatment. According to Lee et al., [13] ketamine has been reported to be effective for the treatment of hypersensitization of central nervous system as the antagonist for NMDA receptor and its intravenous injection or persistent subcutaneous injection were also effective for the treatment of neuralgia following varicella zoster. The treatment effect of a low-power laser therapy has also been reported. As described here, many drugs and various treatment modalities have been introduced. To such an extent, the incurability of this disease hase been indicated.

According to Canon, through his literature "Canon's law of denervation supersensitivity", in cases in which a single factor was desensitized completely or incompletely from the functional chains of neurons, even if a series of factors which were damaged in the chain should not denervated by other nerves or there should be effects which nerve stimulation or inhibitory/excitatory chemical mediators can have, the hypersensitivity of receptors which are present in the areas where the corresponding nerve was innervated would appear. Besides, this author noted that this hypersensitivity was maximally present and it was dwindled to the terminal part. To put this in another way, if the nerve should be damaged, the hypersensitivity of receptors which are present in all the areas where the nerve was innervated under the areas where the nerve 
damage occurred. This implies not only the hypersensitivity of pain receptors but also that of receptors which are responsive to all the chemical mediators. This phenomenon can induce the abnormal nerve functions even in the absence of marked nerve damage. Due to this abnormal nerve function, the hypersensitivity of receptors in the innveration unit occurs. This is a cause of chronic pain or deeply associated with it [14]. According to Gunn, a great number of various types of pain syndrome where there is grossly a lack of the causal relationship might be associated with the development of system which was generated in the denervation supersensitivity, hypersensitive receptors or individual nerve system. In other words, due to the nerve damage, if a specific nerve should fall short of the reference and should not appropriately function, i.e., the status of neuropathy, the corresponding nerve would be hypersensitive or malfunctioned.

A neuropathic pain is referred to a pain which was developed even in the absence of permanent tissue injury or inflammatory findings and then persistently present. Although the causative stimulations cannot be identified, the response was induced and then persistently present. Eventually for any reasons, if the nerve damage should occur, the nerve would be hypersensitive. A neuropathic pain due to the hypersensitivity following the nerve block has focused on the hypersensitivity of structures which were present in the areas where the damaged nerve was innervated. Using the resolution of this hypersensitivity, the results of pain alleviation could be sufficiently obtained.

The areas where Gunn's IMS therapy could be performed representatively include cases in which there was a neuropathic pain due to the hypersensitivity following the nerve block. A stimulatory effect of dry needle which is used for interventional nerve stimulation therapy generates the tissue damage and current of injury. Then, it releases healing soruce of bio-energy and then causes the local hemorrhage. Thus, it induces the synthesis of growth factor originated from plateletspromoting the wound healing. Besides, it stimulates A-delta fiber and olfactory cells in the white matter of spinal cord. Thus, these cells secrete enkephaline and this leads to the suppression of cells forming substantia gelatinosa. Following this, it blocks the stimulation transferring pathway by which the stimulation is transferred to wide dynamic range (WDR) cells in C-fiber which is involved in the chronic pain. Through this effect, the hypersensitivity of nerve block is decreased or removed. Thus, a neuropathic pain is healed $[7,8]$.

We have speculated that the pathogenesis and etiology of pain in patients with $\mathrm{PHN}$ are associated with the hypersensitivity of nerve block. As the most effective treatment method for desensitizing the hypersensitivity, Gunn's IMS therapy was used and the alleviation of symptoms was expected. We performed Gunn's IMS therapy $1-2$ times a week a total of six times in
Table 2. The Changes of VAS Score and Improvement Rate after Gunn's IMS Treatment

\begin{tabular}{ccccccl}
\hline \multirow{2}{*}{ Case no. } & \multicolumn{5}{c}{ Time (week) } \\
\cline { 2 - 7 } & First visit & 1 & 2 & 3 & 4 & \multicolumn{1}{c}{6} \\
\hline 1 & 7 & 5 & 3 & 3 & 3 & $3(57.1 \%)$ \\
2 & $7-8$ & 5 & 4 & 3 & 3 & $2(71.4-75 \%)$ \\
3 & $7-8$ & 4 & 4 & 3 & 3 & $2(57.1-75 \%)$ \\
4 & 8 & 3 & 3 & 2 & 2 & $2(75 \%)$ \\
\hline
\end{tabular}

( ) express improvement rate. VAS: visual analogue scale, Gunn's IMS: Gunn's intramuscular stimulation.

four patients with PHN who visited us with a chief complaint of chest pain. For surgical sites, thoracic multifidus muscle and the tissue around the joint were selected. In regard to the treatment effect, VAS scores were decreased from 7-8 points at the time of outpatient visit to $2-3$ points at the completion of treatment. These results indicate that the pain was decreased and the symptoms were improved. The final improvement rate of VAS was $68 \%$ on average and this corresponded to satisfactory treatment outcomes (Table 2).

Given the above background, in patients with PHN who were refractory to the previous drug treatment and nerve block therapy, we assumed that Gunn's IMS therapy or its concomitant use with previous treatment regimens are recommendable. With the application of Gunn's IMS therapy to the treatment of patients with $\mathrm{PHN}$, we obtained satisfactory treatment outcomes. Here, we report our case with a review of literatures.

\section{References}

1. Hanania MM, Briestein D. Postherpetic neuralgia: a review. Cancer Invest 1997; 15: 165-76.

2. Smith G, Covino BG. Acute pain. London, Butterworths. 1985, p 239.

3. Fields HL, Rowbotham M, Baron R. Postherpetic neuralgia: irritable nociceptors and deafferentation. Neurobiol Dis 1998; 5(4): 209-27.

4. Baron R, Saguer M. Mechanical allodynia in postherpetic neuralgia: evidence for central mechanism depending on nociceptive $\mathrm{C}$-fiber degeneration. Neurology 1995; 45(12 Suppl 8): S63-5.

5. Bonezzi C, Demartini L. Treatment options in postherpetic neuralgia. Acta Neurol Scand Suppl 1999; 173: 25-35.

6. Ogata K, Ngaito M. Blood flow of peripheral nerve effects of dissection, stretching and compression. J Hand Surg Br 1986; 11: 10-4.

7. Cheng SSR. Neurophysiology of electroacupuncture analgesia. In: Scientific bases of acupuncture. Edited by Pomeranz B, Strux G: Dusseldorf, Springer-Verlag. 1989, pp 119-35.

8. Garrison DW, Foreman RD. Decreased activity of spontaneous and noxiously evoked dorsal horn cells during transcutaneous electrical nerve stimulation (TENS). Pain 1994; 58: 309-15. 
9. Lomo T. Stimulation of denervated rat soleus muscle with fast and slow activity patterns induces different expression of acetylcholinesterase molecular forms. J Neurosci 1985; 5: 1180-7.

10. Colding A. The effect of regional sympathetic blocks in the treatment of herpes zoster. Acta Anaesth Scand 1969; 13: 133-41.

11. Winnie AP, Hartwell PW. Relationship between time of treatment of acute herpes zoster with sympathetic blockade and prevention of post-herpetic neuralgia. Reg Anesth 1993; 18: 277-82.

12. Lee DI, Kim DO, Kim KS, Kwon MI, Shin KI, Kang WJ. Gabapentin for the treatment of postherpetic neuralgia. Korean J Pain 1999; 12: 188-90.

13. Lee WY, Lee JH, Hong KH. The effect of an intravenous ketamine infusion on a patient who had suffered from postherpetic nueralgia. Korean J Anesthesiol 2002; 42: 555-7.

14. Culp WJ, Ochoa JL. Abnormal nerves and muscles as impulse generators. New York, Oxford University Press. 1982, pp 21-169.

15. Gunn CC. "prespondylosis" and some pain syndroms following denervation supresensitivity. Spine 1980; 5: 185-92. 